\title{
A Generalized Impure Public Good and Linear Characteristics Model of Green Consumption
}

\author{
Nathan W. Chan \\ Yale University \\ nathan.chan@yale.edu
}

\author{
Matthew J. Kotchen \\ Yale University and NBER \\ matthew.kotchen@yale.edu
}

March 2, 2012

\begin{abstract}
This paper provides a general framework for understanding consumer behavior related to goods and services that may be considered environmentally friendly, ethically produced, fairly traded, or some combination thereof. This paper generalizes the impure public good model and derives its comparative static properties. The model accounts for any number of impure public (green) goods and joint production of any number of both private and public characteristics. The generalization provides a bridge between the impure public good model and the well-known linear characteristics model, both of which are special cases of the model developed here. The results show how demand for green goods and characteristics such as environmental quality depends on wealth, exogenously given levels of public goods, and the technologies of joint production. The effects of changes in technology depend critically on whether jointly produced characteristics are complements or substitutes in consumption. Several of the results are rather counterintuitive and differ in meaningful ways from existing models of impure public goods and linear characteristics. The results also illuminate several reasons for greater caution about whether it is reasonable to assume that green goods and services are necessarily beneficial for the provision of public goods.
\end{abstract}




\section{Introduction}

Consumers have grown increasingly concerned about both private and public dimensions of the goods and services that they buy. The trend is clearly evident in the emergence and growth of markets for goods that are environmentally friendly, ethically produced, and fairly traded. This paper considers how goods based on joint production of private and public characteristics pose new questions for microeconomic theory and the understanding of privately provided public goods. We develop and analyze a model that illuminates the increasing number of trade-offs that consumers face among price, product efficacy, and public characteristics consistent with environmental and social concerns. In doing so, the paper generalizes the impure public good model and derives its comparative static properties, many of which are directly related to the public impacts of environmentally and socially conscious consumption. The generalization also provides a bridge between the impure public good model and the well-known linear characteristics model (Lancaster 1971; Gorman 1980), both of which are special cases of the model developed here.

The basic impure public good model was first developed by Cornes and Sandler (1984, 1994, 1996). Consumers are assumed to obtain utility from a private characteristic and a public characteristic, both of which can be obtained jointly and exclusively from an impure public good. The focus of the model is then on comparative static analysis of demand for the impure public good and thereby characteristics. The applicability of the model for understanding environmentally friendly and socially responsible consumption has been noted by Kotchen $(2005,2006)$, who also extends the model to allow substitutes for the impure public good; that is, the extension accounts for the way that jointly produced characteristics of the impure public good may be available separately as well. For example, with availability of shade-grown coffee, which is based on joint production of coffee and rainforest conservation, consumers generally have additional opportunities to purchase conventional coffee, make donations for rainforest conservation, or both.

A limitation of all the existing impure public good models, however, is that they consider only a single impure public good that generates one private and one pub-

lic characteristic. But the real choice setting that consumers face is typically more expansive. There often exist multiple impure public goods providing different quantities of multiple private and public characteristics. Consider the example of organic 
foods, with the provision of health and taste (private characteristics), along with fewer polluting inputs and possibly greater support of local farming communities (public characteristics). Of course, different brands of organic foods are also available, and they each provide different quantities of the private and public characteristics. Similar features can be used to describe markets ranging from household cleaning products to energy efficient appliances. In many cases, heterogeneity among options comes not only from products themselves, but also from multiple certification standards, with one example being lumber that is certified by either the Forest Stewardship Council or the Sustainable Forestry Initiative.

This paper contributes to the literature with a general framework of environmentally and socially conscious consumption that accounts for any number of impure goods and any number of associated characteristics. Building from a basic model with one impure public good and two characteristics, we expand the setup to include two impure public goods in one case and three characteristics in another. These cases are used to build intuition and highlight results before developing the general model. Beyond providing insight into the comparative statics of "green" goods in particular and impure public goods in general, we are not aware of any other paper that derives comparative static results for the linear characteristics model with such generality. While we return to general applicability of our analysis in the concluding section, we focus throughout the paper on application of the model and results to various green goods and different dimensions of environmental quality.

The analysis yields several new results, some of which we highlight at the outset. First, our simplification of the basic framework reveals that improving the technology of a green good can, quite counterintuitively, decrease demand for the good itself. While previous studies have recognized that improvements in a green good's technology can decrease demand for environmental quality (Kotchen 2005, 2006), the direct implications on demand for the green good itself have not been properly analyzed. Second, when considering how technology changes to a green good affect consumer behavior, it is critical to know which green good is changing, as the results depend on the comparative advantages of goods and can have qualitatively different signs. In particular, the model illuminates how changes in technology are fundamental for understanding changes in demand for green goods, but knowing which good's technology is changing is more fundamental for understanding changes in demand for environmental quality. Third, previous models have found that gross complement conditions 
among characteristics are sufficient to ensure that technology improvements increase demand for environmental quality, but as we will show, the result no longer holds in a more general setting. This finding, among others, provides reason for greater caution about whether it is reasonable to assume that green goods and services are necessarily good for the environment.

\section{Basic model}

We begin with the basic setup in Kotchen (2006) but applied in the context of a representative consumer. The consumer derives utility from characteristics of goods rather than from goods themselves. Preferences are represented by a strictly increasing, strictly quasi-concave, and twice differentiable utility function $U(X, Y)$, where $X$ is a private characteristic and $Y$ is a public characteristic in the sense that it is both nonrival and non-excludable. Continuing with the example of organically grown food, we can think of $X$ as nutrition and $Y$ as a reduction in pollution, perhaps from avoiding the use of pesticides. The consumer is endowed with exogenous wealth $w>0$.

There are three market goods that generate characteristics. A conventional good $c$ generates $X$, a direct donation $d$ generates $Y$, and a green good $g$ generates $X$ and $Y$ jointly. These goods can represent, respectively, conventionally grown food, direct donations to reduce pollution, and organically grown food. The units of $c, d$, and $g$ are chosen such that all prices are normalized to unity. ${ }^{1}$ The units of $X$ and $Y$ are chosen such that one unit of $c$ generates one unit of $X$, and one unit of $d$ generates one unit of $Y$. Let $\alpha, \beta>0$ characterize the green good technology such that one unit of $g$ generates $\alpha$ units of $X$ and $\beta$ units of $Y$.

The consumer's utility maximization problem can be written as

$$
\begin{gathered}
\max _{c, d, g} U(X, Y) \text { subject to } \\
X=c+\alpha g, Y=d+\beta g+\widetilde{Y} \text { and } c+d+g=w,
\end{gathered}
$$

where $\tilde{Y}$ represents the exogenously provided level of the public good that the consumer enjoys. In the special case where $\alpha+\beta=1$, the solution to (1) is not unique

\footnotetext{
${ }^{1}$ It is straightforward to expand the model to account for non-normalized prices. We make the simplifying assumption throughout the paper only to reduce notation and focus on the results of interest.
} 
with respect to the choice of goods, though it is unique with respect to characteristics. The reason is that, in this special case, any bundle of $X, Y>0$ can be obtained at the same cost by an infinite number of $c, d, g$ combinations. Moreover, it is straightforward to verify that if $\alpha+\beta<1$, the consumer will never purchase $g$; and if $\alpha+\beta>1$, the consumer will never choose both $c, d>0$. To focus on the interesting case, therefore, we assume hereafter that $\alpha+\beta>1$, which implies that all goods are viable.

It is useful to rewrite the utility maximization problem with choices over characteristics rather than goods:

$$
\begin{gathered}
\max _{X, Y} U(X, Y) \text { subject to } Y \geq \tilde{Y}, \\
\frac{1-\beta}{\alpha} X+Y \leq w+\tilde{Y} \text { and } X+\frac{1-\alpha}{\beta} Y \leq w+\frac{1-\alpha}{\beta} \tilde{Y} .
\end{gathered}
$$

Figure 1 illustrates the budget frontier that includes two linear facets, with the numbered cases corresponding to the order of the budget constraints in (2). The unique solution to (2) can be expressed as the choice of characteristics as a function of the exogenous parameters: $X^{*}(\Theta)$ and $Y^{*}(\Theta)$, where $\Theta=(\alpha, \beta, w, \tilde{Y})$. To simplify subsequent analysis and keep focus on the primary results of interest, we assume throughout the paper an interior solution, that is, a solution not at a kink or corner of the budget frontier.

At any given solution, it facilitates economic intuition to write the optimal choices of characteristics as implicit demand functions of the form

$$
X^{*}\left(p_{X}^{*}(\Theta), p_{Y}^{*}(\Theta), W^{*}(\Theta)\right) \text { and } Y^{*}\left(p_{X}^{*}(\Theta), p_{Y}^{*}(\Theta), W^{*}(\Theta)\right)
$$

where the notation implies that $p_{X}$ and $p_{Y}$ represent the implicit prices on the facet of the budget frontier containing the optimal solution, and $W$ represents full income, which accounts for the individual's endowment and the value of public-good spillins. In particular, the different possibilities for $\left(p_{X}, p_{Y}, W\right)$ with maximization problem (2), which can be seen from the budget constraints, are $\left(\frac{1-\beta}{\alpha}, 1, w+\tilde{Y}\right)$ or $\left(1, \frac{1-\alpha}{\beta}, w+\right.$ $\left.\frac{1-\alpha}{\beta} \tilde{Y}\right)$, depending upon which facet the solution lies.

We now consider the comparative statics of demand for characteristics. We focus on environmental quality $Y$, but the approach is identical (and in many cases symmetric) if one were interested in the results for $X$. We can also back out the com- 


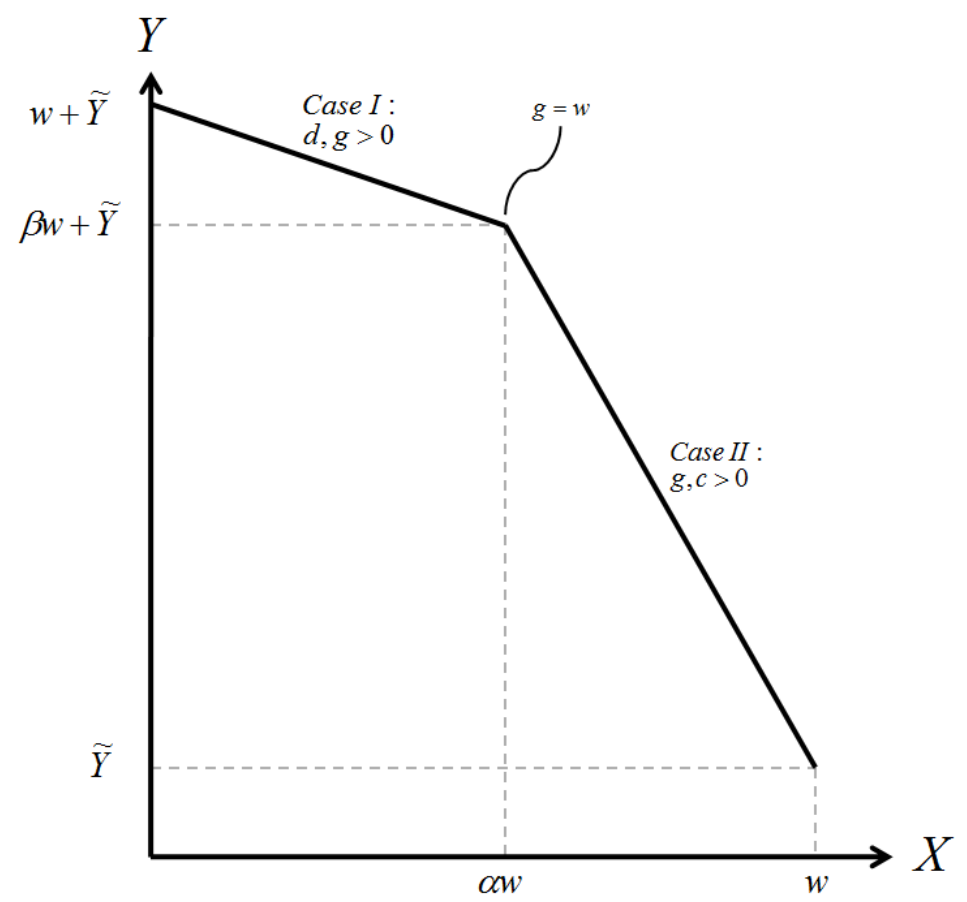

Figure 1: Budget frontier in characteristics space for the basic model 
parative static results for $c, d$, and $g$, though we focus on only the green good $g$ here. Letting $\theta$ denote any one of the exogenous parameters, differentiation of demand for the public characteristic yields

$$
Y_{\theta}^{*}=Y_{p_{X}^{*}}^{*} p_{X \theta}^{*}+Y_{p_{Y}^{*}}^{*} p_{Y \theta}^{*}+Y_{W^{*}}^{*} W_{\theta}^{*}
$$

where subscripts denote partial derivatives. Now substituting in the standard Slutsky decomposition for a change in price and rearranging yields

$$
Y_{\theta}^{*}=\left(\bar{Y}_{p_{X}^{*}}-X^{*} Y_{W^{*}}^{*}\right) p_{X \theta}^{*}+\left(\bar{Y}_{p_{Y}^{*}}-Y^{*} Y_{W^{*}}^{*}\right) p_{Y \theta}^{*}+Y_{W^{*}}^{*} W_{\theta}^{*},
$$

where the new terms $\bar{Y}_{p_{x}^{*}}$ and $\bar{Y}_{p_{y}^{*}}$ are compensated price responses. Expression (4) shows how changes in demand for $Y$ can be understood in terms of familiar substitution and income effects. A change in any one of the parameters may cause a change in the implicit prices, giving rise to the substitution and full-income effects in the first two terms. The third terms captures the fact that a change in the exogenous parameters may also cause a change in full income itself. ${ }^{2}$ The full set of comparative static expressions for $Y$ given a change in each parameter on each facet of the budget frontier is listed and explained in the Appendix. It turns out that if we assume $Y$ is normal with respect to full income, which we do throughout, an immediate result is that $Y_{\theta}^{*}>0$ for $\theta=w, \tilde{Y}^{3}$

The more interesting results occur with respect to changes in the technology parameters $\alpha$ and $\beta$. Because these results have been discussed previously (Kotchen 2005, 2006), albeit with a slightly different setup, we illustrate the basic insights graphically, leaving formal expressions in the Appendix. Consider a technology improvement that increases $\alpha, \beta$, or both. Referring back to Figure 1, this implies a shift in the budget frontier kink point to the northeast. Hence, depending on the facet containing the original solution, the effect is a decrease in the implicit price of either $Y$ or $X$. For the former (case II), demand for $Y$ will always increase; but for the latter, a decrease in the implicit price of $X$ (case I), the sign of the change in demand for $Y$ will depend on whether $Y$ is a gross complement or substitute for $X$. The latter case gives

\footnotetext{
${ }^{2}$ Note that the structure of equations (3) and (4) would be identical if we were considering the comparative statics of demand for $X$ rather than $Y$.

${ }^{3}$ This assumption is not necessary for our derivation of comparative static expressions. It does, however, make clear how several of the counterintuitive results that we will show do not depend on characteristics being inferior.
} 
rise to the counterintuitive possibility that a technology improvement for producing a characteristic, say environmental quality, can decrease demand for that characteristic. With an increase in $\beta$, the consumer substitutes towards more $X$ and less $Y$ by increasing demand for $g$ and decreasing donations $d$. In the case of organic food, the logic would go as follows: an improvement in the environmental benefit of organics causes a consumer to purchase more organics and simultaneously reduce his direct donations to improve environmental quality, with the net result being a decrease in his overall contribution to improving the environment.

An even more counterintuitive result is the fact that a technology improvement for a green good can decrease demand for the good itself. Consider an initial solution on the upper facet and an increase in $\alpha$. It follows that if $Y$ is a gross complement for $X$, then demand for $g$ must decrease. To see this formally, note that we can substitute the budget constraint into the identity for $Y$ to get $Y^{*}(\Theta)=w-g^{*}(\Theta)[1-\beta]+\tilde{Y}$, and differentiating with respect to $\alpha$ yields $g_{\alpha}^{*}=-Y_{\alpha}^{*} /(1-\beta)$, which is negative given the gross complement condition. Intuitively, the change in technology increases demand for $Y$ along with $X$, and the new solution is accomplished by increasing demand for $d$ and decreasing demand $g$. In the example of organics, the story is different than that above: now organic foods become more nutritious which means the consumer can purchase less while maintaining nutrition and freeing up resources to make a larger direct donation. More generally, a necessary condition for this result is that the technology improvement relates to the characteristic that would never be obtained separately on the relevant facet of the budget frontier. In the case discussed, for example, $c=0$ and $X$ is obtained through $g$ only. Importantly, the result that a technology improvement for a green good can decrease demand for the good itself corrects an error in Kotchen (2005). ${ }^{4}$

\section{Two impure public goods}

Now suppose that we extend the model to allow two green goods denoted $g_{1}$ and $g_{2}$, where it continues to hold that $0<\alpha_{k}, \beta_{k}<1$ and $\alpha_{k}+\beta_{k}>1$ for $k=1,2$. These goods can be thought of as two different brands of organic produce or perhaps brands

\footnotetext{
${ }^{4}$ The summary Table 1 in Kotchen (2005) contains the error. For the case of $c$ and $g$, the comparative static sign for $\hat{g}_{\beta}$ should be negative (rather than positive) for the complements column; and for the $d$ and $g$ case, the sign $\hat{g}_{\alpha}$ should be negative (rather than positive) for the complements column.
} 
that conform to two different certification standards. For simplicity let the green good from the previous section satisfy $g=g_{1}$ and hence $\alpha=\alpha_{1}$ and $\beta=\beta_{1}$. Without loss of generality, we assume that $\beta_{1}>\beta_{2}$. This implies that $\alpha_{1}<\alpha_{2}$ is a necessary condition for $g_{2}$ to be viable, but it is not sufficient. Referring back to Figure 1, the assumptions thus far imply that the kink where $g_{2}=w$ is to the southeast of the kink where $g_{1}=w$. But to make $g_{2}$ viable it must be the case that the new kink point lies outside the facet corresponding to case II. It is straightforward to verify that a sufficient condition is $\left(1-\alpha_{2}\right) / \beta_{2}<\left(1-\alpha_{1}\right) / \beta_{1}$. This implies that when consuming $g_{2}$ and $c$, the ratio of $p_{Y} / p_{X}$ must be less than when consuming $g_{1}$ and $c$. Similar reasoning is necessary to ensure that $g_{1}$ continues to be viable, and this condition is $\alpha_{1} /\left(1-\beta_{1}\right)<\alpha_{2} /\left(1-\beta_{2}\right)$, which implies that when consuming $g_{1}$ and $d$, the ratio $p_{Y} / p_{X}$ is less than when consuming $g_{2}$ and $d$.

The consumer's utility maximization problem is now written as

$$
\begin{gathered}
\max _{c, d, g_{1}, g_{2}} U(X, Y) \text { subject to } \\
X=c+\alpha_{1} g_{1}+\alpha_{2} g_{2}, Y=d+\beta_{1} g_{1}+\beta_{2} g_{2}+\widetilde{Y} \text { and } c+d+g_{1}+g_{2} \leq w .
\end{gathered}
$$

Rewriting the problem in characteristics space yields

$$
\begin{gathered}
\max _{X, Y} U(X, Y) \text { subject to } Y \geq \tilde{Y} \text { and } p_{X} X+p_{Y} Y \leq W \text { where } \\
\left(p_{X}, p_{Y}, W\right)=\left\{\begin{array}{l}
\left(\frac{1-\beta_{1}}{\alpha_{1}}, 1, w+\tilde{Y}\right), \\
\left(\frac{\beta_{1}-\beta_{2}}{\eta}, \frac{\alpha_{2}-\alpha_{1}}{\eta}, w+\frac{\alpha_{2}-\alpha_{1}}{\eta} \tilde{Y}\right), \\
\left(1, \frac{1-\alpha_{2}}{\beta_{2}}, w+\frac{1-\alpha_{2}}{\beta_{2}} \tilde{Y}\right)
\end{array}\right\} \text { and } \eta=\beta_{1} \alpha_{2}-\beta_{2} \alpha_{1} .
\end{gathered}
$$

Figure 2 illustrates the budget frontier, which has three facets (cases I, II, and III) corresponding in order with the different sets of implicit prices and full income in (6). For this problem, the implicit prices are based on the slope of the facets and are straightforward to derive. In Section 5, we describe a general method of solving for implicit prices for this version of the model and others. In parallel with the previous section, and given a solution to (6), we can define the implicit demand function for the public characteristic as $Y^{*}\left(p_{X}^{*}(\Theta), p_{Y}^{*}(\Theta), W^{*}(\Theta)\right)$, where the vector of exogenous parameters now includes additional terms: $\Theta=\left(\alpha_{1}, \alpha_{2}, \beta_{1}, \beta_{2}, w, \widetilde{Y}\right)$.

Even with two green goods, the general expression for comparative static results is identical to equation (4) from the previous section. We again derive explicit results 


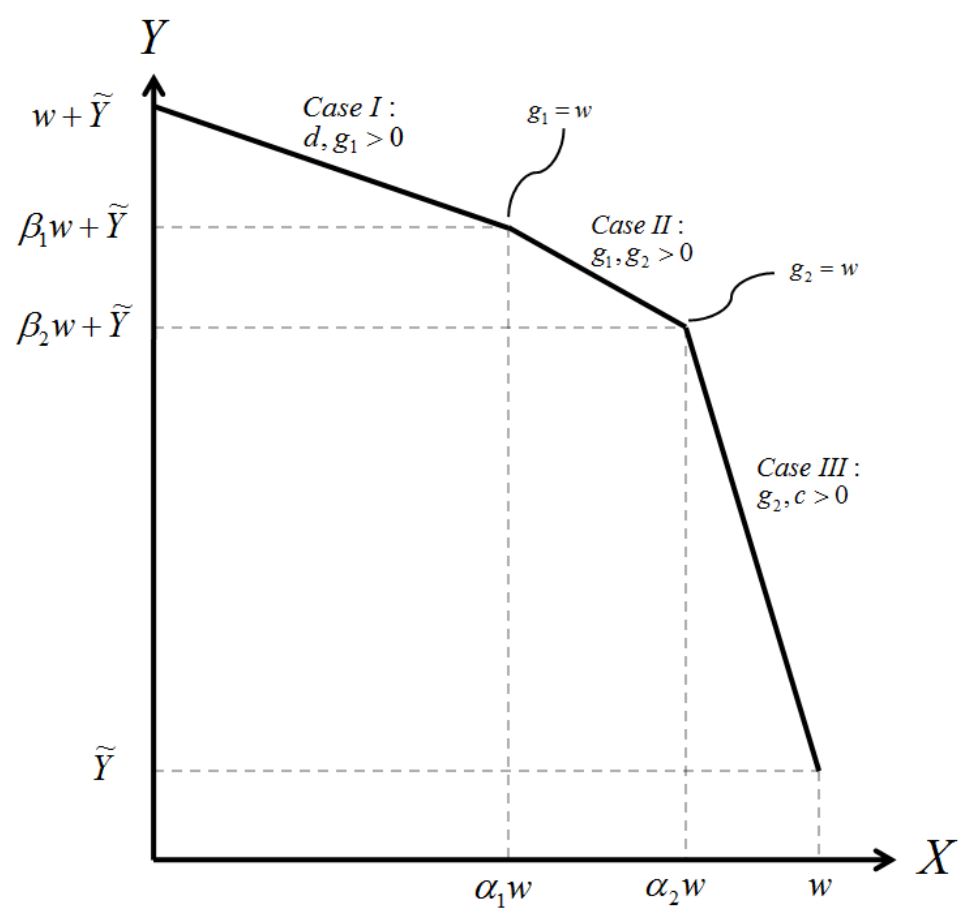

Figure 2: Budget frontier in two dimensional characteristics space with two green goods 
for each parameter and include them in the Appendix, while here we continue to discuss the different and important results intuitively, with a focus on the technology parameters.

Some results are nearly identical to those shown previously. The upper and lower facets (cases I and III) have the same comparative static properties as the upper and lower facets from the previous section. The only difference is they apply only for changes to the corresponding green good's technology. That is, changes in $g_{1}$ affect the upper facet, while changes in $g_{2}$ affect the lower facet. Despite the similar structure of these results, one difference when there is more than one green good is that changing the technology of one good need not affect consumer behavior, as consumers may already have chosen a consumption bundle on a different facet. This is not possible when there is only one green good.

The middle facet, where $g_{1}, g_{2}>0$, is where the comparative statics differ most importantly, but the results can still be understood in terms of changes in the relative implicit prices. Consider first a change in the technology of $g_{1}$ - the green good that has a comparative advantage in producing the public characteristic. ${ }^{5}$ It follows that both $Y_{\alpha_{1}}^{*}>0$ and $Y_{\beta_{1}}^{*}>0$, and intuition follows from the fact that a technology improvement on either $\alpha_{1}$ or $\beta_{1}$ shifts the upper kink point to the northeast, causing a decrease in the implicit price of $Y$ (along with a full-income adjustment) on the middle facet. In contrast, technology improvements that increase either $\alpha_{2}$ or $\beta_{2}$ will decrease the implicit price of $X$ (along with a full income adjustment) on the middle facet, and the change in demand for $Y$ will again depend on whether it is a gross complement or substitute for $X$. If $Y$ is a gross substitute for $X$, it follows that $Y_{\alpha_{2}}^{*}<0$ and $Y_{\beta_{2}}^{*}<0$. Note that these results imply that technological improvements to a green good will decrease demand for environmental quality.

An important result, however, is that the gross complement condition is no longer sufficient to ensure an increase in demand for $Y$. While shown formally in the Appendix, the intuition follows from recognizing the points rationing nature of the model's setup, as this is a case where it makes a qualitative difference. ${ }^{6}$ On the middle facet,

\footnotetext{
${ }^{5} \mathrm{On}$ a given facet, we say that a good has a comparative advantage in producing a characteristic if it provides that characteristic at least cost. We can likewise describe that good as being the most efficient provider of that characteristic. We will use these terms interchangeably.

${ }^{6}$ Generically, the problem here has been studied in other contexts as a points rationing problem, for which price changes are subject to additional rationing constraints (e.g., Tobin 1952; McManus 1954). In the case studied here, the additional rationing constraint arises from the fact that characteristics must be obtained via goods, which have fixed prices.
} 
we have discussed how an increase in either $\alpha_{2}$ or $\beta_{2}$ decreases the implicit price of $X$, but the way we typically think about price changes is somewhat different because of the budget frontier's pivot at the other kink point anchored by $g_{1}$. Because the kink point is not on the $Y$-axis, there is effectively an increase in the price of $Y$ as well that is captured through a full income adjustment. Hence changes in demand for $Y$ will depend on the incentives created by a decrease in the price of $X$ and a simultaneous increase in the price of $Y$. Assuming $Y$ is a gross complement for $X$ creates incentives that push in opposite directions, which is the source of the indeterminate sign of $Y_{\alpha_{2}}^{*}$ and $Y_{\beta_{2}}^{*}$ on the middle facet. ${ }^{7}$ This is not, however, the case with a gross substitute condition, under which we know that demand for $Y$ must decrease.

The result that a technology improvement for a green good can decrease demand for the good itself continues to hold, though we gain further insight in the model with two green goods. On the middle facet, the binding budget constraint is $g_{1}^{*}+g_{2}^{*}=w$ and by definition $Y^{*}=\beta_{1} g_{1}^{*}+\beta_{2} g_{2}^{*}+\tilde{Y}$. Substituting $g_{1}^{*}$ out of the second equation and differentiating yields $g_{2 \alpha_{2}}^{*}=-Y_{\alpha_{2}}^{*} /\left(\beta_{1}-\beta_{2}\right)$, which can be negative only if $Y$ is a gross complement for $X$. Following the same steps but using the identity that $X^{*}=$ $\alpha_{1} g_{1}^{*}+\alpha_{2} g_{2}^{*}$ yields $g_{1 \beta_{1}}^{*}=-X_{\beta_{1}}^{*} /\left(\alpha_{2}-\alpha_{1}\right)$, which can be negative only if $X$ is a gross complement for $Y$. Combining these two cases reveals a more general result about when a technology improvement will decrease demand for a green good: it occurs when (a) the technology relates to the characteristic for which the good has a comparative advantage and (b) the other characteristic is a gross complement. ${ }^{8}$ Intuitively, the insight is that making an efficient green good more efficient enables substitution towards other characteristics, which in turn, and counterintuitively, reduces demand for the green good itself. This result underscores how the comparative statics for a green good depend critically upon which technology parameter changes.

By considering two green goods, we also learn that the relative positioning of goods plays an important role in how demand for environmental quality responds to changes in technology. In contrast to the results for goods, the comparative statics of demand for characteristics $X$ and $Y$ depend critically on which good is being altered, rather than on which technology parameter changes. That is, with more than one

\footnotetext{
${ }^{7}$ The Appendix shows how the sign of the result is indeterminate in general, but we also construct a numerical example in which demand for $Y$ decreases.

${ }^{8}$ Note that this generalizes insights from the previous section about decreases in demand for $g$. In that case, $g$ has a comparative advantage in $X$ on the upper facet; therefore, when $Y$ is a gross complement for $X$, an improvement in $\alpha$ decreases demand for $g$.
} 
green good, it is critical to know which good's technology parameters are changing to ascertain the effect on demand for $Y$. This is especially true since, as we have shown, the results can have qualitatively different signs, which arises because improvements to the two goods have opposite effects on implicit prices.

A final and related observation is that changes in the technology of one good can render another inviable. If, for example, $\Delta \alpha_{1}>\alpha_{2}-\alpha_{1}>0$, then $g_{2}$ would no longer be viable, as $g_{1}$ would be more efficient at producing both characteristics, and the model would return to having one green good. While we eliminate this possibility as long as our viability assumptions continue to hold, a remark about the potential to "knock out" goods in this fashion is nevertheless worthwhile.

\section{Three characteristics}

We now return to having a single green good but consider the effect of having three characteristics, two of which are public. Continuing with the example of organic food, the new public characteristic could be the support of local farming, in addition to the characteristics of nutrition and reduced pollution. Let $Y^{A}$ and $Y^{B}$ denote the two public characteristics, which can be obtained jointly through $g$ with technologies $\beta^{A}$ and $\beta^{B}$ and separately through $d^{A}$ and $d^{B}$. Note that consumers can support local farming by purchasing locally grown organic food, making a direct donation, or both. Prices remain normalized to unity, and all other features of the setup are the same. But now to maintain viability of all four market goods and study the interesting case, we assume $0<\alpha, \beta^{A}, \beta^{B}<1$ and $\alpha+\beta^{A}+\beta^{B}>1 .^{9}$

The consumer's utility maximization problem can be written as

$$
\begin{gathered}
\max _{c, d^{A}, d^{B}, g} U\left(X, Y^{A}, Y^{B}\right) \text { subject to } \\
X=c+\alpha g, Y^{A}=d^{A}+\beta^{A} g+\tilde{Y}^{A}, Y^{B}=d^{B}+\beta^{B} g+\tilde{Y}^{B} \\
\text { and } c+d^{A}+d^{B}+g \leq w .
\end{gathered}
$$

Note that the setup here is a more general version of one with two private character-

\footnotetext{
${ }^{9}$ To maintain the most interesting case, in which there is a third characteristics to consider, the viability assumption made here is stronger than necessary. Strictly speaking, viability of all goods requires that the first inequality satisfy $0 \leq \alpha, \beta^{A}, \beta^{B}<1$ with at least two parameters strictly greater than zero.
} 
istics and one public characteristic, whereby the only modification would be to set either of the two spillin amounts, $\tilde{Y}^{A}$ or $\tilde{Y}^{B}$, to zero. Following the steps in previous sections, we again rewrite the problem with choices over characteristics:

$$
\begin{gathered}
\max _{X, Y^{A}, Y^{B}} U\left(X, Y^{A}, Y^{B}\right) \text { subject to } Y^{A} \geq \tilde{Y}^{A}, Y^{B} \geq \tilde{Y}^{B}, \\
\text { and } p_{X} X+p_{Y^{A} Y^{A}+p_{Y^{B}} Y^{B} \leq W \text { where }} \\
\left(p_{X}, p_{Y^{A}}, p_{Y^{B}}, W\right)=\left\{\begin{array}{c}
\left(\frac{1-\beta^{A}-\beta^{B}}{\alpha}, 1,1, w+\tilde{Y}^{A}+\tilde{Y}^{B}\right), \\
\left(1,1, \frac{1-\alpha-\beta^{A}}{\beta^{B}}, w+\tilde{Y}^{A}+\frac{1-\alpha-\beta^{A}}{\beta^{B}} \tilde{Y}^{B}\right), \\
\left(1, \frac{1-\alpha-\beta^{B}}{\beta^{A}}, 1, w+\frac{1-\alpha-\beta^{B}}{\beta^{A}} \tilde{Y}^{A}+\tilde{Y}^{B}\right)
\end{array}\right\} .
\end{gathered}
$$

The budget frontier, as illustrated in Figure 3, lies within the three-dimensional characteristics space and consists of three facets (planes denoted as cases I, II, and III) defined respectively by the different sets of implicit prices and full income. For this scenario and others, the implicit prices can be derived based on the gradients of facets, but as mentioned previously, we describe a more systematic and general way of solving for implicit prices in the next section. Given the unique solution for $Y^{h *}(\Theta)$ for $h=A, B$ and $\Theta=\left(\alpha, \beta^{A}, \beta^{B}, w, \tilde{Y}^{A}, \tilde{Y}^{B}\right)$, the more familiar demand functions for the public characteristics can be written as $Y^{h *}\left(p_{X}^{*}(\Theta), p_{Y^{A}}^{*}(\Theta), p_{Y^{B}}^{*}(\Theta), W^{*}(\Theta)\right)$ for $h=A, B$. Differentiating with respect to any of the exogenous parameters $\theta$ and substituting in the Slutsky equation yields the general expression for comparative static results for both of the public characteristics:

$Y_{\theta}^{h *}=\left(\bar{Y}_{p_{X}^{*}}^{h}-X^{*} Y_{W^{*}}^{h *}\right) p_{X \theta}^{*}+\left(\bar{Y}_{p_{Y A}^{*}}^{h}-Y^{A *} Y_{W^{*}}^{h *}\right) p_{Y^{A} \theta}^{*}+\left(\bar{Y}_{p_{Y}^{*}}^{h}-Y^{B *} Y_{W^{*}}^{h *}\right) p_{Y^{B} \theta}^{*}+Y_{W^{*}}^{h *} W_{\theta}^{*}$.

The difference between (4) and (9) is the additional term on account of there being a price for the second public characteristic. The complete set of results is again included in the Appendix, while here we focus on fundamental insights associated with changes in the green good's technology.

It is useful to recognize that on each facet of the budget frontier, $g$ has a comparative advantage in providing one characteristic and therefore pins down the corresponding implicit price. It follows that improvements in any of the green good's technologies, which will push out the kink point where $g=w$, will decrease the implicit price of the characteristic for which $g$ has a comparative advantage and hence increase demand for that characteristic. Focusing on $Y^{A}$, this reasoning ap- 


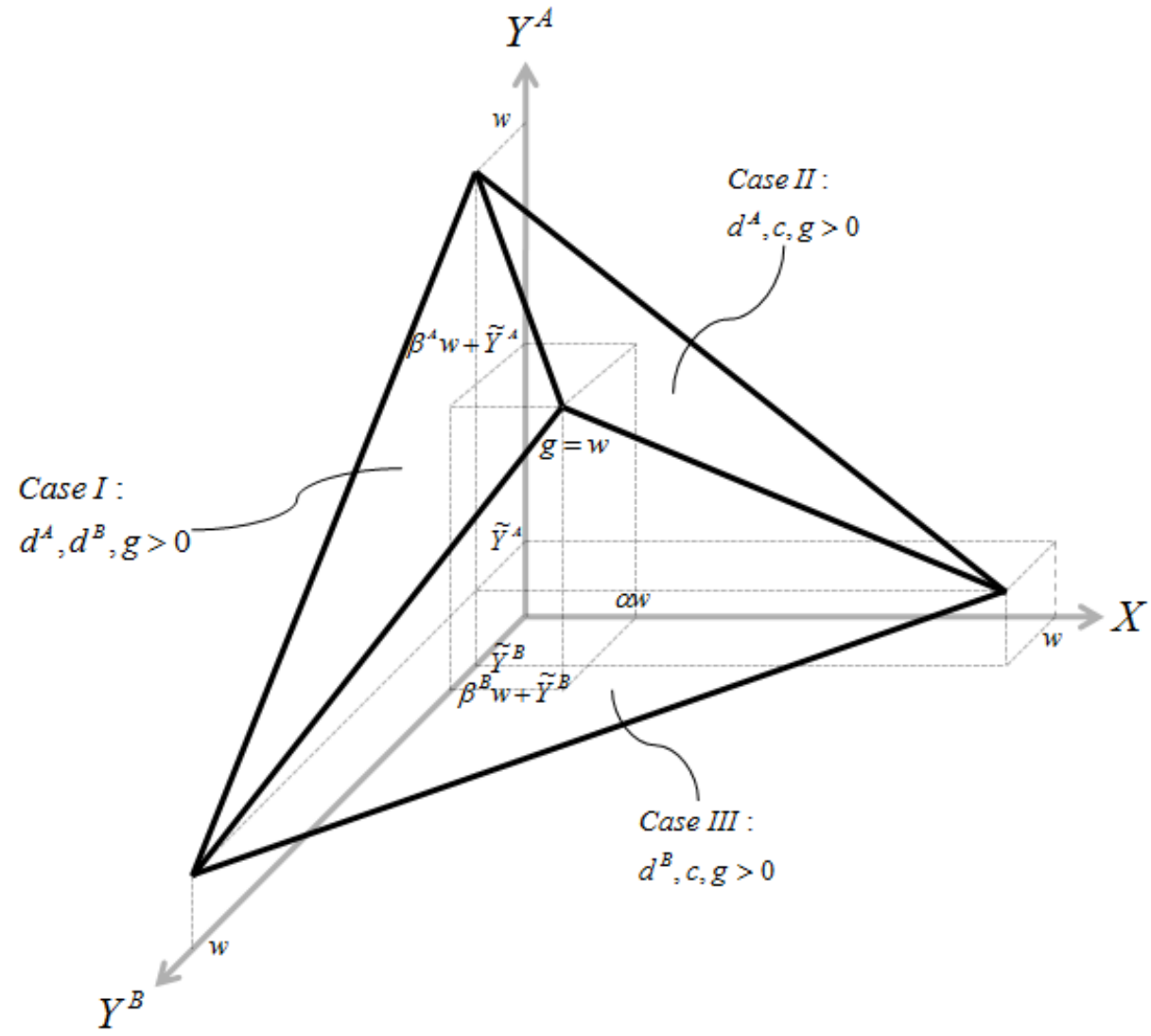

Figure 3: Budget frontier in characteristics space with one green good and three characteristics 
plies to any consumption bundle in case III, implying that, for this case, $Y_{\theta}^{A *}>0$ for $\theta=\alpha, \beta^{A}, \beta^{B}$. Note that this result is a generalization of the insight made previously for two green goods in two-dimensional characteristics space, where we found that improving the technology for the more environmentally friendly, green good along a facet led to unambiguous increases in demand for $Y$ on that facet.

Other results with a similar intuition to those shown previously are that on the facet for case I, changes in demand for $Y^{A}$ will depend on whether $Y^{A}$ is a gross complement or substitute for $X$. The same holds on the facet for case II, depending on the complementarity or substitutability of $Y^{A}$ for $Y^{B}$. It is the case, however, that the points rationing nature of the problem leaves the sign of the result indeterminate with respect to the gross complement condition. With three characteristics, there is an additional and important result: the possibility for net complements exists and, as shown in the Appendix, this produces an unambiguous result that differs from the previous sections but has the same intuition. In this case, a net complement condition $\left(Y^{A}\right.$ for $\left.Y^{B}\right)$ is sufficient to ensure that improvements in a green good's technology increase demand for the environmental public good $Y^{A}$.

Consideration of three characteristics produces further new results. Even when the effects of technology changes on demand for one characteristic are clear, the same changes can increase demand for both of the other characteristics, decrease demand for both, or increase demand for one and increase demand for the other. Not surprisingly, the different results depend on the cross-price effects on demand, and there is greater flexibility when there are more than two characteristics. Importantly, this implies that technology improvements that increase demand for one jointly produced public good can decrease demand for another. When it comes to green goods, for example, increasing demand for one dimension of environmental quality can decrease demand for other jointly produced public goods, including, perhaps, other dimensions of environmental quality. In the context of our motivating example, this implies that changes in the technology of organic food can have different consequences on the implicit demand for environmental quality and local farming.

This same intuition underlies the way that demand for $g$ can still decrease in response to an improvement in its technology. Consider a consumption bundle consistent with case I, where $d^{A}, d^{B}, g>0$. Substituting the budget constraint into the identity for $Y^{A}$ yields $Y^{A *}=w-d^{B *}-\left(1-\beta^{A}\right) g^{*}$. Differentiating and simplifying yields $g_{\alpha}^{*}=-\left(Y_{\alpha}^{A *}+Y_{\alpha}^{B *}\right) /\left(1-\beta^{A}-\beta^{B}\right)$. It follows that $g_{\alpha}^{*}<0$ if both public char- 
acteristics are gross complements for $X$. Consistent with that from previous sections, the intuition is that increasing $\alpha$ decreases the implicit price of $X$ which stimulates demand for $X$ and, because of the gross complement assumption, for $Y^{A}$ and $Y^{B}$ as well. But the increase in $\alpha$ means that the consumer can satisfy this demand by decreasing demand for $g$ and increasing demand for $d^{A}$ and $d^{B}$. One feature that differs with multiple characteristics, however, is that it remains possible for $g_{\alpha}^{*}<0$ if only $Y^{A}$ or only $Y^{B}$ is a gross complement for $X$, assuming the complementarity is sufficiently strong.

\section{$5 \quad$ Full generalization}

Building on intuition developed in the preceding sections, we now fully generalize the model to account for any number of green goods and any number of jointly produced private and public characteristics. In doing so, we also dispense with the assumption that private and public characteristics are available separately through pure private and pure public goods (i.e., $c$ and $d$ ), as these are simply special cases of a green (or more generally, impure public) good without joint production.

Consumer preferences are specified over $N$ private characteristics and $M$ public characteristics, represented by $U\left(X^{1}, \ldots, X^{N}, Y^{1}, \ldots, Y^{M}\right)$. The goods available to the consumer are $g_{k}$ for $k=1, \ldots, K$, the number of goods satisfies $K \geq N+M$, and the technology of each good $k$ is characterized by the vector of parameters $v_{k}=$ $\left(\alpha_{k}^{1}, \ldots, \alpha_{k}^{N}, \beta_{k}^{1}, \ldots, \beta_{k}^{M}\right)$. We continue to assume prices are normalized to unity and $w$ denotes the consumer's exogenous endowment.

We also continue to assume that all goods are viable. To derive the viability condition, let $G$ denote the set of $K$ goods, and we know that each $v_{k}$ represents a point in $N+M$-space. Now define

$$
\operatorname{Co}(G)=\left\{\sum_{k=1}^{K} \gamma_{k} v_{k}: \forall \gamma_{k} \geq 0 \text { such that } \sum_{k=1}^{K} \gamma_{k}=1\right\}
$$

which is a convex hull in characteristics space based on the set of $K$ goods. ${ }^{10}$ Viability of each $g_{k}$ thus requires that its corresponding $v_{k}$ defines an extreme point and that there does not exist $s \in C o(G)$ such that $s>v_{k}$. The latter part of this condition

\footnotetext{
${ }^{10}$ Note that $C o(G)$ represents the set of characteristic allocations obtainable under the assumptions that $w=1$, income is fully spent, and $\tilde{Y}^{m}=0$ for all $m$.
} 
ensures that we are considering the part of the frontier furthest from the origin. Together, these conditions ensure that each $v_{k}$ defines at least one facet on the frontier of $C o(G)$ and for each of these facets, its corresponding $g_{k}$ is the most efficient provider of a single characteristic, and is therefore viable.

With this formulation, the consumer's maximization problem can be written as

$$
\begin{gathered}
\max _{g_{1}, \ldots, g_{K}} U\left(X^{1}, \ldots, X^{N}, Y^{1}, \ldots, Y^{M}\right) \text { subject to } \sum_{k=1}^{K} g_{k} \leq w \\
{\left[\begin{array}{c}
X^{1} \\
\vdots \\
X^{N}
\end{array}\right]=\left[\begin{array}{ccc}
\alpha_{1}^{1} & \cdots & \alpha_{K}^{1} \\
\vdots & \ddots & \vdots \\
\alpha_{1}^{N} & \cdots & \alpha_{K}^{N}
\end{array}\right]\left[\begin{array}{c}
g_{1} \\
\vdots \\
g_{K}
\end{array}\right], \text { and }} \\
{\left[\begin{array}{c}
Y^{1} \\
\vdots \\
Y^{M}
\end{array}\right]=\left[\begin{array}{ccc}
\beta_{1}^{1} & \cdots & \beta_{K}^{1} \\
\vdots & \ddots & \vdots \\
\beta_{1}^{M} & \cdots & \beta_{K}^{M}
\end{array}\right]\left[\begin{array}{c}
g_{1} \\
\vdots \\
g_{K}
\end{array}\right]+\left[\begin{array}{c}
\tilde{Y}^{1} \\
\vdots \\
\tilde{Y}^{M}
\end{array}\right]}
\end{gathered}
$$

Following the steps shown previously, we can rewrite the consumer's problem with choices over characteristics. Doing so in a familiar format, however, requires solving for the implicit prices on each facet of the budget frontier. We first state the problem and then describe how to solve for the implicit prices. The utility maximization problem can be rewritten as

$$
\begin{gathered}
\max _{X^{1}, \ldots, X^{N}, Y^{1}, \ldots, Y^{M}} U\left(X^{1}, \ldots, X^{N}, Y^{1}, \ldots, Y^{M}\right) \text { subject to } Y^{m} \geq \tilde{Y}^{m} \text { for all } m \\
\text { and } p_{X^{1}} X^{1}+\ldots+p_{X^{N}} X^{N}+p_{Y^{1}} Y^{1}+\ldots+p_{Y^{M}} Y^{M} \leq W \text { for } \\
\left\{p_{X^{1}}^{f}, \ldots, p_{X^{N}}^{f}, p_{Y^{1}}^{f}, \ldots, p_{Y^{M}}^{f}, W^{f}\right\}_{f=1}^{F} .
\end{gathered}
$$

There are $F$ sets of implicit prices and full income, representing the number of facets on the budget frontier. Each facet is defined by $N+M$ goods and therefore has $N+M-1$ dimensions. In general, the budget frontier is the frontier of $C o(G)$ dilated by $w$ and translated for spillins.

The task now is to verify that we can solve for $\left(p_{X^{1}}, \ldots, p_{X^{N}}, p_{Y^{1}}, \ldots, p_{Y^{M}}, W\right)$ corresponding with each of the $F$ facets. We know that $W=w+p_{Y^{1}} \tilde{Y}^{1}+\ldots+p_{Y^{M}} \tilde{Y}^{M}$, and subtracting the value of spillins from each side of the binding budget constraint 
yields

$$
p_{X^{1}} X^{1}+\ldots+p_{X^{N}} X^{N}+p_{Y^{1}}\left(Y^{1}-\tilde{Y}^{1}\right)+\ldots+p_{Y^{M}}\left(Y^{M}-\tilde{Y}^{M}\right)=w .
$$

We thus have $N+M$ unknown prices for each facet. For any given facet, we can use the technologies of the goods that define its extreme points, which yields $N+M$ equations of the form

$$
\left[\begin{array}{cccccc}
\alpha_{1}^{1} w & \cdots & \alpha_{1}^{N} w & \beta_{1}^{1} w & \cdots & \beta_{1}^{M} w \\
\vdots & \ddots & & & & \vdots \\
\vdots & & \ddots & & & \vdots \\
\vdots & & & \ddots & & \vdots \\
\vdots & & & & \ddots & \vdots \\
\alpha_{N+M}^{1} w & \cdots & \alpha_{N+M}^{N} w & \beta_{N+M}^{1} w & \cdots & \beta_{N+M}^{M} w
\end{array}\right]\left[\begin{array}{c}
p_{X^{1}} \\
\vdots \\
p_{X^{N}} \\
p_{Y^{1}} \\
\vdots \\
p_{Y^{M}}
\end{array}\right]=\left[\begin{array}{c}
w \\
\vdots \\
w \\
w \\
\vdots \\
w
\end{array}\right] .
$$

It is then straightforward to solve for the implicit prices for each facet using Cramer's Rule.

Example 1 To illustrate, we use the approach to solve for the implicit prices on the two facets of the basic model in Section 2. Each case in Figure 1 represents a facet, and the analogs to equation (11) are

$$
\begin{aligned}
& {\left[\begin{array}{cc}
0 & w \\
\alpha w & \beta w
\end{array}\right]\left[\begin{array}{l}
p_{X} \\
p_{Y}
\end{array}\right]=\left[\begin{array}{l}
w \\
w
\end{array}\right] \text { for case } I,} \\
& {\left[\begin{array}{cc}
\alpha w & \beta w \\
w & 0
\end{array}\right]\left[\begin{array}{l}
p_{X} \\
p_{Y}
\end{array}\right]=\left[\begin{array}{l}
w \\
w
\end{array}\right] \text { for case II. }}
\end{aligned}
$$

Then applying Cramer's Rule yields the implicit prices shown previously.

To characterize the solution to (10), let $\vec{Z}=\left(X^{1}, \ldots, X^{N}, Y^{1}, \ldots, Y^{M}\right)$, where we index the elements of $Z$ with the superscript $l$. The complete solution can thus be written as $Z^{l *}(\Theta)$ for $l=1, \ldots, N+M$, and expressing the solution in terms of more familiar demand functions, we can write

$$
Z^{l *}\left(p_{X^{1}}^{*}(\Theta), \ldots, p_{X^{N}}^{*}(\Theta), p_{Y^{1}}^{*}(\Theta), \ldots, p_{Y^{M}}^{*}(\Theta), W^{*}(\Theta)\right)
$$


The comparative static results on a facet for any characteristic $l$ are thus

$$
\frac{\partial Z^{l *}}{\partial \theta}=\sum_{n=1}^{N} \frac{\partial Z^{l}}{\partial p_{X^{n}}^{*}} \frac{\partial p_{X^{n}}^{*}}{\partial \theta}+\sum_{m=1}^{M} \frac{\partial Z^{l}}{\partial p_{Y^{m}}^{*}} \frac{\partial p_{Y^{m}}^{*}}{\partial \theta}+\frac{\partial Z^{l *}}{\partial W^{*}} \frac{\partial W^{*}}{\partial \theta} .
$$

Substituting in the Slutsky decomposition and using more compact notation yields

$$
Z_{\theta}^{l *}=\sum_{n=1}^{N}\left(\bar{Z}_{P_{X}^{*}}^{l}-X^{n *} Z_{W^{*}}^{l *}\right) p_{X^{n} \theta}^{*}+\sum_{m=1}^{M}\left(\bar{Z}_{p_{Y}^{*}}^{l}-Y^{m *} Z_{W^{*}}^{l *}\right) p_{Y^{m} \theta}^{*}+Z_{W^{*}}^{l *} W_{\theta}^{*} .
$$

This expression generalizes all of the results we have shown previously. It also makes clear how, in general, changes in demand depend on the implicit cross-price effects among private characteristics, public characteristics, and across both. Moreover, when changes in exogenous parameters affect implicit prices, there are additional income effects to consider on account of points rationing constraints and the value of spillins. Finally, as we have shown for particular cases, changes in demand for characteristics, such as those shown in (12) can be used to derive changes in demand for goods themselves.

\section{Conclusion}

Using environmentally friendly goods and services as a motivating application, this paper generalizes the impure public good model and derives its comparative static properties. While other models consider a single impure public good and joint production of two characteristics, our model accounts for any number of impure public goods and joint production of any number of both private and public characteristics. The generalization captures as special cases the standard impure public good model and the well-known linear characteristics model, the comparative static properties of which, to our knowledge, have never been shown with such generality. The results are important in terms of understanding how demand for characteristics, and thereby goods and services, depends on wealth, exogenously given levels of public goods, and most importantly, the technologies of joint production.

Markets for green goods and services are a natural application of the model, and we show how the effect of changes in technology depend fundamentally on whether jointly produced characteristics are complements or substitutes in consumption. Some 
results are rather counterintuitive, namely that improvements in the technology of a green good can decrease demand for environmental quality and for the green good itself. While similar results have been shown before, we find that the conditions under which they occur differs in meaningful ways with more than one impure public good and two characteristics. The impure public good model has also been used to study topics in environmental policy (Rübbelke 2002) and common-pool resources (Vicary 2009, 2011). But, even in these cases, there is often more than one dimension of environmental quality and/or private benefits and costs to consider, and the model developed here provides a basis for taking these important features into account.

More generally, our results should be of interest to researchers in other fields of economics as well. Within public economics, the impure public good model has been used in various ways to understand charitable giving. Many of the models posit different private motives (i.e., private characteristics) that people consider when making donations, ranging from warm glow (Andreoni 1989, 1990), social approval (Hollander 1990), prestige (Harbaugh 1998), and signalling about income (Glazer and Konrad 1996). It is certainly the case, however, that several motives can operate simultaneously, requiring a more general framework of the type we develop here. Finally, within industrial organization, the linear characteristics model provides the basis for studying consumer choice and estimating fundamental parameters such as cross-price elasticities in continuous hedonic choice models (e.g., Chan 2006). In such cases, the model that we develop provides a more complete theoretical underpinning for interpreting the array of results that are possible. 


\section{Appendix}

\subsection{Basic setup}

The comparative static results for a change in each of the exogenous parameters are the following, where the cases refer to those indicated in Figure 1 :

- $Y_{w}^{*}=Y_{W^{*}}^{*}>0$,

- $Y_{\tilde{Y}}^{*}=\left\{\begin{array}{ll}Y_{W^{*}}>0 & \text { if case I } \\ \frac{1-\alpha}{\beta} Y_{W^{*}}>0 & \text { if case II }\end{array}\right.$,

- $Y_{\alpha}^{*}= \begin{cases}-\frac{1-\beta}{\alpha^{2}}\left(\bar{Y}_{p_{X}^{*}}-X^{*} Y_{W^{*}}^{*}\right) & \text { if case I } \\ -\frac{1}{\beta}\left(\bar{Y}_{p_{Y}^{*}}-Y^{*} Y_{W^{*}}^{*}+\tilde{Y} Y_{W^{*}}^{*}\right)>0 & \text { if case II }\end{cases}$

- $Y_{\beta}^{*}=\left\{\begin{array}{ll}-\frac{1}{\alpha}\left(\bar{Y}_{p_{X}^{*}}-X^{*} Y_{W^{*}}^{*}\right) & \text { if case I } \\ -\frac{1-\alpha}{\beta^{2}}\left(\bar{Y}_{p_{Y}^{*}}-Y^{*} Y_{W^{*}}^{*}+\tilde{Y} Y_{W^{*}}^{*}\right)>0 & \text { if case II }\end{array}\right.$.

The normality assumption implies $Y_{w}^{*}, Y_{\tilde{Y}}^{*}>0$. We know $Y_{\alpha}^{*}, Y_{\beta}^{*}>0$ if case II (i.e., $c>0$ ) because increases in the technology parameters decrease the implicit price of $Y$ causing an increase in demand. In case I (i.e., $d>0$ ), the signs depend on a further condition because the same changes in technology will decrease the implicit price of $X$. It follows that $Y_{\alpha}^{*}$ and $Y_{\beta}^{*}$ in case I are positive (negative) when $Y$ is a gross complement (substitute) for $X$.

\subsection{Two impure public goods}

To simplify notation, let $\phi=\alpha_{2}-\alpha_{1}$ and $\psi=\beta_{1}-\beta_{2}$, in addition to $\eta=\beta_{1} \alpha_{2}-\beta_{2} \alpha_{1}$. Note that $\eta, \phi, \psi>0$. The comparative static results can be written as follows, where the cases refer to those indicated in Figure 2:

- $Y_{w}^{*}=Y_{W^{*}}^{*}>0$,

- $Y_{\tilde{Y}}^{*}=\left\{\begin{array}{ll}Y_{W^{*}}^{*}>0 & \text { if case I } \\ \frac{\phi}{\eta} Y_{W^{*}}^{*}>0 & \text { if case II } \\ \frac{1-\alpha_{2}}{\beta_{2}} Y_{W^{*}}^{*}>0 & \text { if case III }\end{array}\right.$, 
- $Y_{\alpha_{1}}^{*}= \begin{cases}-\frac{1-\beta_{1}}{\alpha_{1}^{2}}\left(\bar{Y}_{p_{X}^{*}}-X^{*} Y_{W^{*}}^{*}\right) & \text { if case I } \\ \frac{\beta_{2} \psi}{\eta^{2}}\left(\bar{Y}_{p_{X}^{*}}-X^{*} Y_{W^{*}}^{*}\right)-\frac{\alpha_{2} \psi}{\eta^{2}}\left(\bar{Y}_{p_{Y}^{*}}-Y^{*} Y_{W^{*}}^{*}+\tilde{Y} Y_{W^{*}}^{*}\right)>0 & \text { if case II } \\ 0 & \text { if case III }\end{cases}$

- $Y_{\beta_{1}}^{*}=\left\{\begin{array}{ll}-\frac{1}{\alpha_{1}}\left(\bar{Y}_{p_{X}^{*}}-X^{*} Y_{W^{*}}^{*}\right) & \text { if case I } \\ \frac{\beta_{2} \phi}{\eta^{2}}\left(\bar{Y}_{p_{X}^{*}}-X^{*} Y_{W^{*}}^{*}\right)-\frac{\alpha_{2} \phi}{\eta^{2}}\left(\bar{Y}_{p_{Y}^{*}}-Y^{*} Y_{W^{*}}^{*}+\tilde{Y} Y_{W^{*}}^{*}\right)>0 & \text { if case II , } \\ 0 & \text { if case III }\end{array}\right.$,

- $Y_{\alpha_{2}}^{*}= \begin{cases}0 & \text { if case I } \\ -\frac{\beta_{1} \psi}{\eta^{2}}\left(\bar{Y}_{p_{X}^{*}}-X^{*} Y_{W}^{*}\right)+\frac{\alpha_{1} \psi}{\eta^{2}}\left(\bar{Y}_{p_{Y}^{*}}-Y^{*} Y_{W}^{*}+\tilde{Y} Y_{W}^{*}\right) & \text { if case II } \\ -\frac{1}{\beta_{2}}\left(\bar{Y}_{p_{Y}^{*}}-Y^{*} Y_{W}^{*}+\tilde{Y} Y_{W}^{*}\right)>0 & \text { if case III }\end{cases}$

- $Y_{\beta_{2}}^{*}=\left\{\begin{array}{ll}0 & \text { if case I } \\ -\frac{\beta_{1} \phi}{\eta^{2}}\left(\bar{Y}_{p_{X}^{*}}-X^{*} Y_{W^{*}}^{*}\right)+\frac{\alpha_{1} \phi}{\eta^{2}}\left(\bar{Y}_{p_{Y}^{*}}-Y^{*} Y_{W^{*}}^{*}+\tilde{Y} Y_{W^{*}}^{*}\right) & \text { if case II } \\ -\frac{1-\alpha_{2}}{\left(\beta_{2}\right)^{2}}\left(\bar{Y}_{p_{Y}^{*}}-Y^{*} Y_{W^{*}}^{*}+\tilde{Y} Y_{W^{*}}^{*}\right)>0 & \text { if case III }\end{array}\right.$.

Note that for cases I and III (i.e., where $d>0$ or $c>0$, respectively), the results are isomorphic to those previously derived for the basic model. For case II (i.e., where $\left.g_{1}, g_{2}>0\right)$, the results are different. It is straightforward to verify that $Y_{\alpha_{1}}^{*}, Y_{\beta_{1}}^{*}>$ 0 . This follows because, after several steps of rearranging terms, one can see that satisfying $\alpha_{2}\left(Y^{*}-\tilde{Y}\right)>\beta_{2} X^{*}$ is sufficient to sign both results, and the inequality must hold for all quantities $\left(X^{*}, Y^{*}\right)$ consistent with case II. Intuition for these results is that, for example, an increase in either parameter decreases the implicit price of $Y$. It also holds that $Y_{\alpha_{2}}^{*}$ and $Y_{\beta_{2}}^{*}$ will always have the same sign and will be negative if $Y$ is a gross substitute for $X$.

Importantly, we see in this case that assuming a gross complement condition is not sufficient to ensure that the signs are positive. The reason is due to the points rationing nature of the problem. While, for example, an increase in either parameter decreases the implicit price of $X$, the pivot on the other kink point pinned down by $g_{1}$ implies a simultaneous increase in the price of $Y$ that is captured in a full income adjustment. Hence, with changes in the technologies of $g_{2}$, the two price changes push demand for $Y$ in opposite directions, resulting in an indeterminate sign; whereas the two price effects push demand for $Y$ in the same direction with a gross substitute condition or with changes in the technologies of $g_{1}$.

We construct a numerical example to verify that, even with the gross complement condition and a reasonable functional form of the utility function, it is possible for an 
improvement in a $g_{2}$ technology to decrease demand for $Y$. Preferences are given by the CES utility function $U(X, Y)=\left(.5 X^{r}+.5 Y^{r}\right)^{1 / r}$ where $r=(s-1) / s$ and $s=.9$. The endowment is $w=1, \tilde{Y}=0$, and the initial technology parameters are $\alpha_{1}=.1$, $\beta_{1}=.95, \alpha_{2}=.95$, and $\beta_{2}=.1$. Now changing $\alpha_{2}$ to $\alpha_{2}^{\prime}=.96$, it is straightforward to verify that the example produces the desired result: $Y_{\alpha_{2}}^{*}<0$ with $Y$ being a gross complement for $X$.

\subsection{Multiple Characteristics}

Below are the comparative static results for the market scenario with one green good and three characteristics, two of which are public goods. We report the results for $Y^{A}$ only, as all expressions are symmetric for $Y^{B}$. The cases refer to those referenced in Figure 3.

- $Y_{w}^{A *}=Y_{W^{*}}^{A *}>0$,

- $Y_{\tilde{Y}^{A}}^{A *}=\left\{\begin{array}{ll}Y_{W^{*}}^{A *}>0 & \text { if case I } \\ Y_{W^{*}}^{A *}>0 & \text { if case II } \\ \frac{1-\alpha-\beta^{B}}{\beta^{A}} Y_{W^{*}}^{A *}>0 & \text { if case III }\end{array}\right.$,

- $Y_{\tilde{Y}^{B}}^{A *}= \begin{cases}Y_{W^{*}}^{A *}>0 & \text { if case I } \\ \frac{1-\alpha-\beta^{A}}{\beta^{B}} Y_{W^{*}}^{A *}>0 & \text { if case II } \\ Y_{W^{*}}^{A *}>0 & \text { if case III }\end{cases}$

- $Y_{\alpha}^{A *}=\left\{\begin{array}{ll}-\frac{1-\beta^{A}-\beta^{B}}{\alpha^{2}}\left(\bar{Y}_{p_{x}^{*}}^{A}-X^{*} Y_{W^{*}}^{A *}\right) & \text { if case I } \\ -\frac{1}{\beta^{B}}\left(\bar{Y}_{p_{Y}^{*}}^{A}-Y^{B *} Y_{W^{*}}^{A *}+\tilde{Y}^{B} Y_{W^{*}}^{A *}\right) & \text { if case II } \\ -\frac{1}{\beta^{A}}\left(\bar{Y}_{Y_{Y A}^{*}}^{A}-Y^{A *} Y_{W^{*}}^{A *}+\tilde{Y}^{A} Y_{W^{*}}^{A *}\right)>0 & \text { if case III }\end{array}\right.$,

- $Y_{\beta^{A}}^{A *}= \begin{cases}-\frac{1}{\alpha}\left(\bar{Y}_{p_{X}^{*}}^{A}-X^{*} Y_{W^{*}}^{A *}\right) & \text { if case I } \\ -\frac{1}{\beta^{B}}\left(\bar{Y}_{p^{*}}^{A}-Y^{B *} Y_{W^{*}}^{A *}+\tilde{Y}^{B} Y_{W^{*}}^{A *}\right) & \text { if case II } \\ -\frac{1-\alpha-\beta^{B}}{\left(\beta^{A}\right)^{2}}\left(\bar{Y}_{p_{Y}^{*}}^{A}-Y^{A *} Y_{W^{*}}^{A *}+\tilde{Y}^{A} Y_{W^{*}}^{A *}\right)>0 & \text { if case III }\end{cases}$

- $Y_{\beta^{B}}^{A *}=\left\{\begin{array}{ll}-\frac{1}{\alpha}\left(\bar{Y}_{p_{X}^{*}}^{A}-X^{*} Y_{W^{*}}^{A *}\right) & \text { if case I } \\ -\frac{1-\alpha-\beta^{A}}{\left(\beta^{B}\right)^{2}}\left(\bar{Y}_{p_{Y}^{*}}^{A}-Y^{B *} Y_{W^{*}}^{A *}+\tilde{Y}^{B} Y_{W^{*}}^{A *}\right) & \text { if case II } \\ -\frac{1}{\beta^{A}}\left(\bar{Y}_{p_{Y}^{*}}^{A}-Y^{A *} Y_{W^{*}}^{A *}+\tilde{Y}^{A} Y_{W^{*}}^{A *}\right)>0 & \text { if case III }\end{array}\right.$. 
The effect of changes in any technology are positive for case III. As discussed in the main text, this is due to the fact that on the facet where $d^{B}, c, g>0$, the impure public good is the most efficient way to obtain $Y^{A}$. Improving the technology of the green good thus decreases the implicit price of $Y^{A}$ and stimulates demand. The results for case I (i.e., when $d^{A}, d^{B}, g>0$ ) follow a familiar pattern because changes in the green technology affect the implicit price of $X$. Hence if $Y^{A}$ is a gross complement (substitute) for $X$, the sign of each expression is positive (negative).

The expressions for case II (i.e., when $d^{A}, c, g>0$ ) have unambiguous signs and are negative if $Y^{A}$ is a gross substitute for $Y^{B}$, the price of which changes with changes in the green good's technology. In this case, however, the sign of the results remain ambiguous if $Y^{A}$ is a gross complement for $Y^{B}$, with the reason being similar to that described in the previous section, due to the points rationing feature of the setup. Nevertheless, there is an important difference: if the two public characteristics are net complements, the sign of the expressions is positive, but note that net complements was not a possibility in the previous scenario, where there are only two characteristics in the model's setup. For with preferences specified over only two goods (or characteristics as in the model here), convex preferences require a relationship of net substitutes. 


\section{References}

Andreoni, J. (1989) Giving With Impure Altruism: Applications to Charity and Ricardian Equivalence. Journal of Political Economy, 97:1447-1458.

Andreoni, J. (1990). Impure Altruism and Donations to Public Goods: A Theory of Warm-Glow Giving. Economic Journal, 100:464-77.

Chan, T. Y. (2006) Estimating a Continuous Hedonic-Choice Model with an Application to Demand for Soft Drinks. RAND Journal of Economics, 37:466-482.

Cornes, R. and T. Sandler (1984) Easy Riders, Joint Production, and Public Goods. Economic Journal, 94:580-598.

Cornes, R. and T. Sandler (1994) The Comparative Static Properties of the Impure Public Good Model. Journal of Public Economics, 54:403-421.

Cornes, R. and T. Sandler (1996) The Theory of Externalities, Public Goods and Club Goods, Second Edition. Cambridge University Press.

Glazer, A, and K. A. Konrad (1986) A Signalling Explanation for Charity. American Economic Review, 86:1019-1028.

Gorman, W. M. (1980) A Possible Procedure for Analyzing Quality Differentials in the Egg Market. The Review of Economic Studies, 47:843-856.

Harbaugh, W. T. (1998). What Do Donations Buy? A Model of Philanthropy Based On Prestige and Warm Glow. Journal of Public Economics, 67:269-84.

Hollander, H. (1990). A Social Exchange Approach To Voluntary Cooperation. American Economic Review, 80:1157-1167.

Kotchen, M. J. (2005) Impure Public Goods and the Comparative Statics of Environmentally Friendly Consumption. Journal of Environmental Economics and Management, 49:281-300.

Kotchen, M. J. (2006) Green Markets and Private Provision of Public Goods. Journal of Political Economy, 114:816-834. 
Lancaster, K. (1971) Consumer Demand: A New Approach. New York: Columbia University Press.

McManus, M. (1954) The Geometry of Points Rationing. The Review of Economic Studies, 22:1-14.

Rübbelke, D. (2002) International Climate Policy to Combat Global Warming: An Analysis of the Ancillary Benefits of Reducing Carbon Emissions. Edward Elgar Publishing, Cheltenham.

Tobin, J. (1952) A Survey of the Theory of Rationing. Econometrica, 20:521-553.

Vicary, S. (2009) The Voluntary Provision of a Public Good in an International Commons. Canadian Journal of Economics, 42:984-996.

Vicary, S. (2011) Public Goods and the Commons: a Common Approach. Journal of Public Economic Theory, 13:47-69. 